\title{
Performance Analysis on Different Modulation Techniques of MIMO in Multipath Fading Channel
}

\author{
Nidhi Raghav ${ }^{1}$, Veeranna.D ${ }^{2}$ \\ ${ }^{1}$ (M.Tech student, Dept .Electronics and communication, Lingaya University, India) \\ ${ }^{2(}$ Asst Prof. Dept. Electronics and communication, Lingaya University, India)
}

\begin{abstract}
This paper presents performance comparison of various modulation techniques in Rayleigh fading channel. In the comparison, the space time coding has been used. The performance comparison are made on the basis of bit error rate(BER) and symbol error rate(SER) performance matrices over Rayleigh fading channel with BPSK,QPSK,8PSK and QAM modulation technique. The comparison results shows that in Rayleigh fading the bit error rate and symbol error rate is least for QAM modulation technique as compared to other three modulation techniques i.e. BPSK, QPSK and 8PSK.
\end{abstract}

Keywords - BER, MIMO, modulation techniques, Rayleigh fading, SER

\section{INTRODUCTION}

Multiple-input multiple-output (MIMO) is a promising technology for next generation wireless systems to enhance capacity and robustness of the link. MIMO technology is enabled by the presence of multiple transmit antennas and multiple receive antennas in the communication link and is being investigated for cellular communication. The benefits of MIMO communication are obtained through a combination of antenna arrays that provide spatial diversity from the propagation channel. Space-time coding is a specific method to make use of multiple antennas from a diversity point of view improving link reliability and power efficiency [3]. In general, space-time codes are able to obtain a full diversity order of with Rate $=1$. From an "error rate performance view", space-time coding brings diversity advantage (i.e. slope of the performance curve) and coding advantages (i.e. horizontal shift in the curve). Since a MIMO channel has input-output relationship $\mathrm{y}=\mathrm{Hx}+\mathrm{n}$, the symbol transmitted over the channel each symbol time is a vector rather than a scalar, as in traditional modulation for the SISO channel.

\section{SYSTEM MODEL}

Consider a wireless communication system with two antennas at the base station and one antenna at the receiver (decoder). At each time instant $t$, signals are transmitted simultaneously from the two transmit antennas. The channel is assumed to be a flat fading channel and it can be relaxed without any change to the method of encoding and decoding. At the receiver one antenna is present where decoding process is carried out as shown.

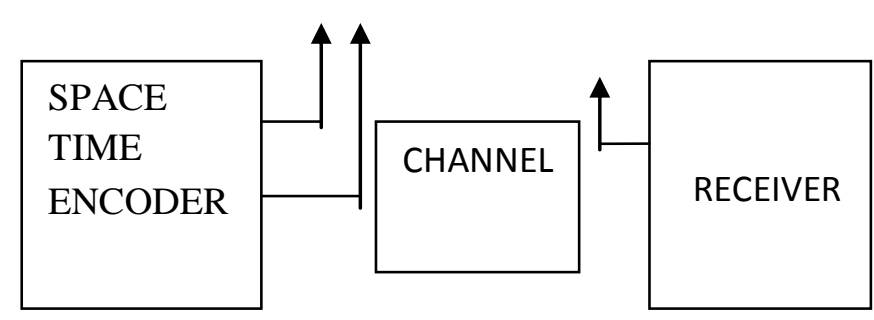

Figure 1 System Model
Figure-2 shows the baseband representation of the new two branch transmit diversity scheme. The scheme uses two transmit antennas and one receive antenna and is called as Alamouti's code which is the first open-loop transmit diversity technique which provides full diversity with linear processing at the receiver complexity[1]. The new transmit diversity scheme can improve the error performance, data rate and capacity of wireless communications systems. The decreased sensitivity to fading may allow the use of higher level modulation schemes to increase the effective data rate, or smaller reuse factors in a multi cell environment to increase system capacity. The scheme may also be used to increase the range or the coverage area of wireless systems. In other words, the new scheme is effective in all of the applications where system capacity is limited by multipath fading and, hence, may be a simple and cost-effective way to address the market demands for quality and efficiency without a complete redesign of existing systems. Furthermore, the scheme seems to be a superb candidate for next-generation wireless systems as it effectively reduces the effect of fading at the remote units using multiple transmit antennas at the base stations. It may be defined by the following three functions

- The encoding and transmission sequence of information symbols at the transmitter;

- The combining scheme at the receiver;

- The decision rule for maximum likelihood detection.

A. The Encoding and Transmission Sequence: At a given symbol period, two signals are simultaneously transmitted from the two antennas. The signal transmitted from antenna zero is denoted by $s_{0}$ and from antenna one by $s_{1}$. During the next symbol period signal $\left(-\mathrm{s}_{1} *\right)$ is transmitted from antenna zero, and signal $\mathrm{s}_{0} *$ is transmitted from antenna one where * shows the complex conjugate operation. This sequence is shown in table 2.1. In table 2.1, the encoding is done in space and time (space-time coding). The encoding, however, may also be done in space and frequency. Instead of two adjacent symbol periods, two adjacent 
carriers may be used (space-frequency coding).
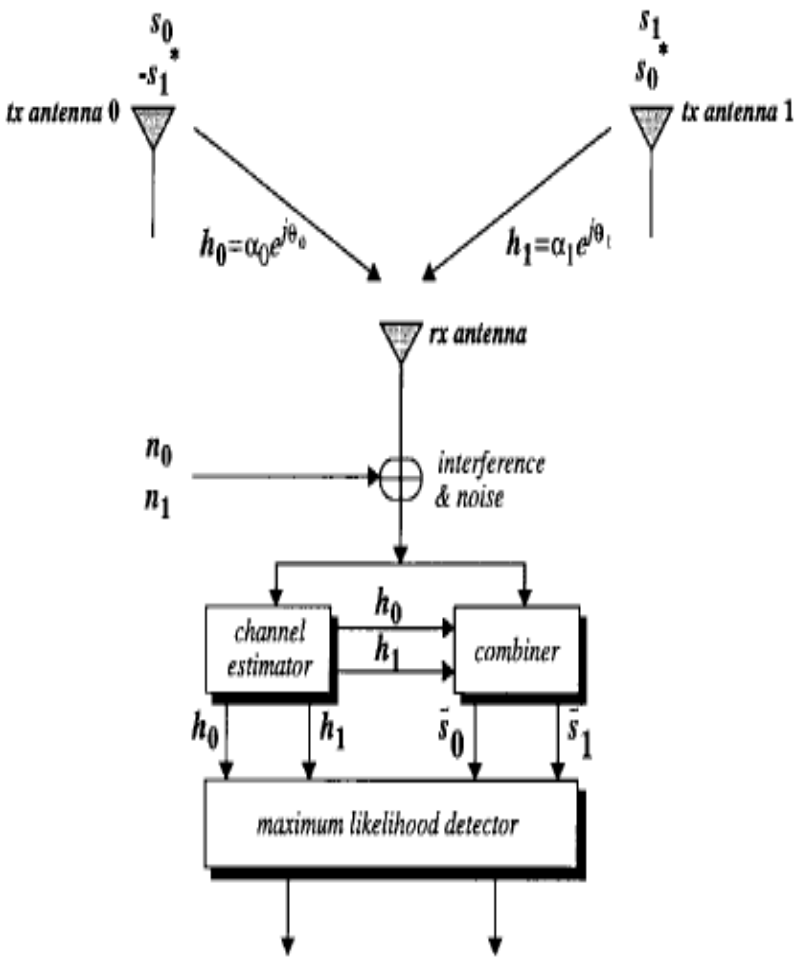

Figure-2: The new two-branch transmit diversity scheme with one receiver [1]

Table-2.1 Encoding and transmission sequence for the two branch transmit diversity scheme

\begin{tabular}{|l|l|l|l|}
\hline \multicolumn{1}{|l|}{ Time } & \multicolumn{1}{|l|}{ Space } & \\
& & $\begin{array}{l}\text { Antenna } \\
0\end{array}$ & $\begin{array}{l}\text { Antenna } \\
1\end{array}$ \\
\hline Time $\mathbf{t}$ & & $\mathrm{S}_{0}$ & $\mathrm{~S}_{1}$ \\
\hline Time $\mathbf{t}+\mathbf{T}$ & $\left(-\mathrm{S}_{1}^{*}\right)$ & $\mathrm{S}_{0}^{*}$ \\
\hline
\end{tabular}

The channel at time may be modeled by a complex multiplicative distortion $\mathrm{h}_{0}(\mathrm{t})$ for transmit antenna zero and $\mathrm{h}_{1}$ (t) for transmit antenna one. Assuming that fading is constant across two consecutive symbols, we can write

$h_{0}(t)=h_{0}(t+T)=h_{0}=a_{0} e^{j \theta_{0}}$

(1) $h_{1}(t)=h_{1}(t+T)=h_{1}=a_{1} e^{j \theta_{1}}$

(2)

$\mathrm{T}$ symbol duration the received

signals can then be expressed as

$$
\begin{aligned}
& r_{0}(t)=r(t)=h_{0} s_{0}+h_{1} s_{1}+n_{0} \\
& r_{1}=r_{1}(t+T)=-h_{0} s_{1}^{*}+h_{1} s_{0}^{*}+n_{1}
\end{aligned}
$$

where $r_{0}$ and $r 1$ are the received signals at time $t$ and $(t+T)$ and $\mathrm{n}_{0}$ and $\mathrm{n}_{1}$ are complex random variables representing receiver noise and interference.
B. The Combining Scheme: The combiner shown in Figure- .2 builds the following two combined signals that are sent to the maximum likelihood detector:

$$
\begin{aligned}
& \tilde{s}_{0}=h_{0}^{*} r_{0}+h_{1} r_{1}^{*} \\
& \tilde{s}_{1}=h_{1}^{*} r_{0}-h_{0} \\
& \tilde{s}_{0}=\left(\alpha_{0}^{2}+\alpha_{1}^{2}\right) s_{0}+h_{0}^{*} n_{0}+h_{1} n_{1}^{*} \\
& \tilde{s}_{1}=\left(\alpha_{0}^{2}+\alpha_{1}^{2}\right) s_{1}-h_{0} n_{1}^{*}+h_{1}^{*} n_{0}
\end{aligned}
$$

C. The Maximum Likelihood Decision Rule: These combined signals are then sent to the maximum likelihood detector which, for each of the signals and, uses the decision rule expressed for PSK signals. The resulting combined signals in (7) and (8) are equivalent to that obtained from two-branch MRRC. The only difference is phase rotations on the noise components which do not degrade the effective SNR. Therefore, the resulting diversity order from the new two-branch transmit diversity scheme with one receiver is equal to that of two-branch MRRC.

\section{CHANNEL MODEL}

Multipath fading is due to the constructive and destructive combination of randomly delayed, reflected, scattered and diffracted signal components. This type of fading is relatively fast and is therefore responsible for the short-term signal variations. Depending on the nature of the radio propagation environment, there are different models describing the statistical behavior of the multipath-fading envelope. The Rayleigh is the most commonly used statistical models to represent small-scale fading phenomenon.

Rayleigh Fading Channel: The Rayleigh distribution is commonly used to describe the statistical time varying nature of the received envelope of a flat fading signal, or the envelope of an individual multipath component [5]. In the Rayleigh flat fading channel model, it is assumed that the channel induces amplitude, which varies in time according to the Rayleigh distribution. When the channel impulse response is modeled as a zero-mean complexvalued Gaussian process, the envelope at any instant is Rayleigh-distributed. The Rayleigh distribution of a received complex envelope of a signal $z(t)=|r(t)|$ at any time $\mathrm{t}$ is given as

$$
p_{z}(x) \frac{x}{\sigma^{2}} e^{\left(\frac{-x^{2}}{2 \sigma^{2}}\right)} \quad(x \geq 0)
$$




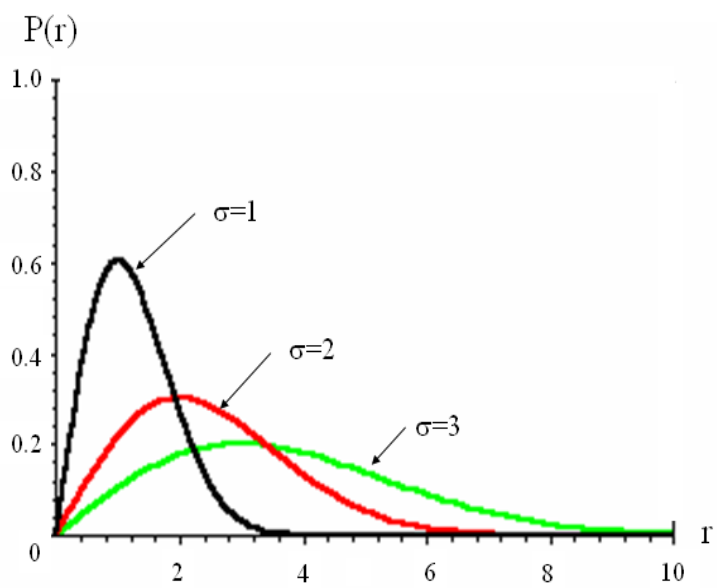

Figure-3: PDF of the envelope variation

Where $\sigma$ is the root man square value of the received voltage signal before envelope detection and $\sigma^{2}$ is the timeaverage power of the received signal before envelope detection. It is well known that the envelope of the sum of two quadrature Gaussian noise signals obeys a Rayleigh distribution. This fading distribution could be applied to any scenario where there is no LOS path between transmitter and receiver antennas.

\section{MODULATION TECHNIQUES}

A modulation scheme is needed to transmit information over a communication channel. Among the various modulation methods are; amplitude modulation (data encoded by changing the amplitude of the signal), frequency modulation (data encoded by changing the frequency of the signal), and phase modulation (data encoded by changing the phase of the signal).

The modulation methods chosen for this paper are: Binary phase shift keying (BPSK), Quadrature phase shift keying (QPSK), M-ary phase shift keying (8PSK) and MQuadrature amplitude modulation (M-QAM).

\section{RESULTS}

SIMULATION RESULTS OF BER FOR RAYLEIGH FADING WITH DIFFERENT MODULATION TECHNIQUE

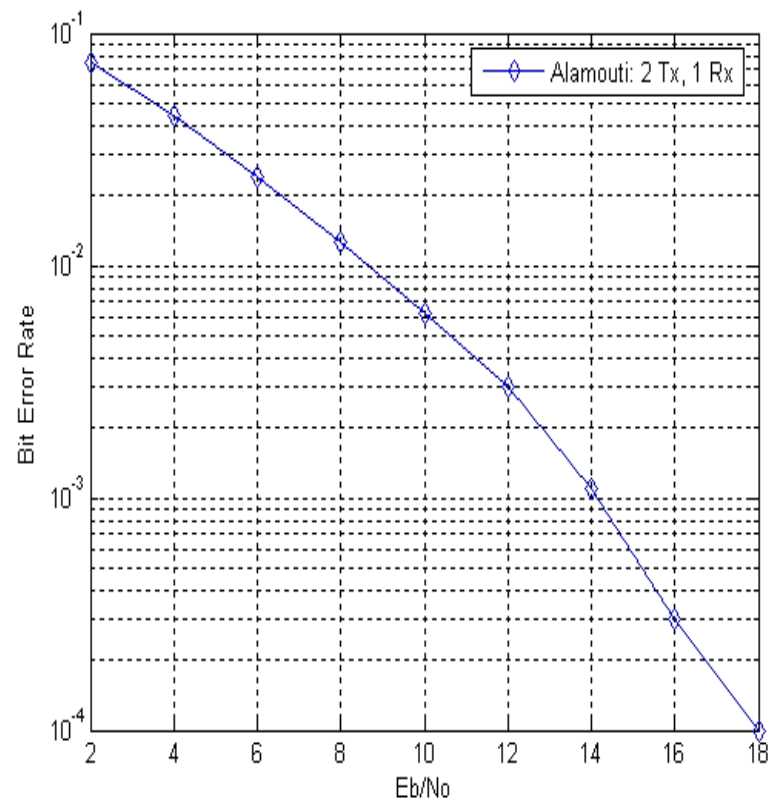

Figure-4: BER performance of BPSK for Rayleigh fading

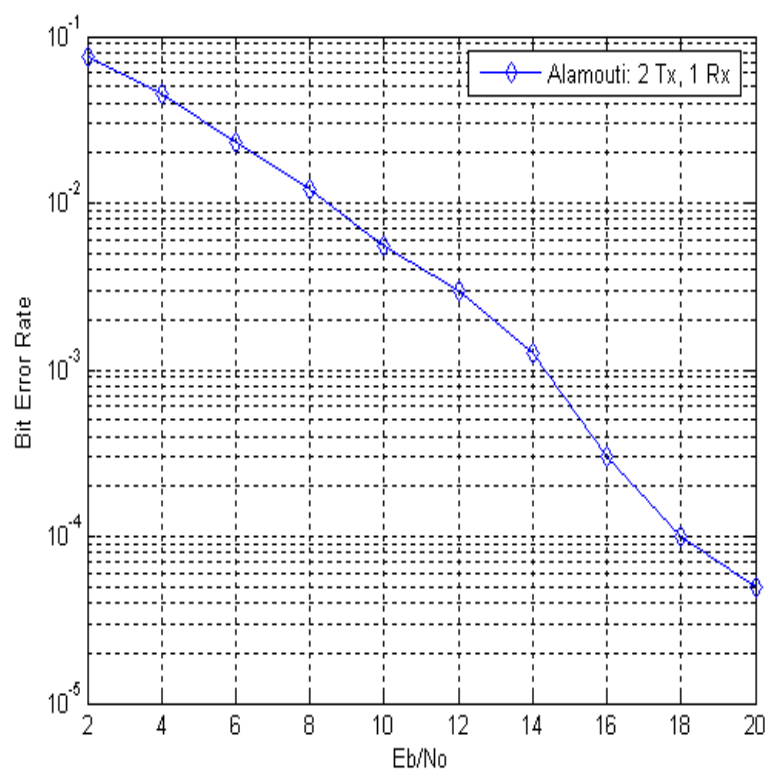

Figure-5: BER performance of QPSK for Rayleigh fading. 


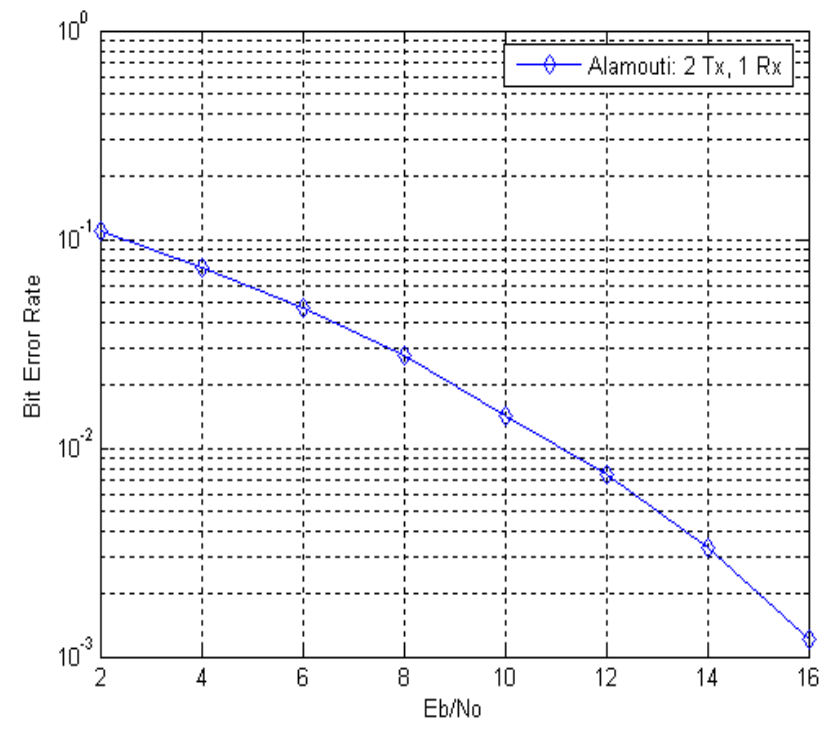

Figure-6: BER performance of 8-PSK for Rayleigh fading

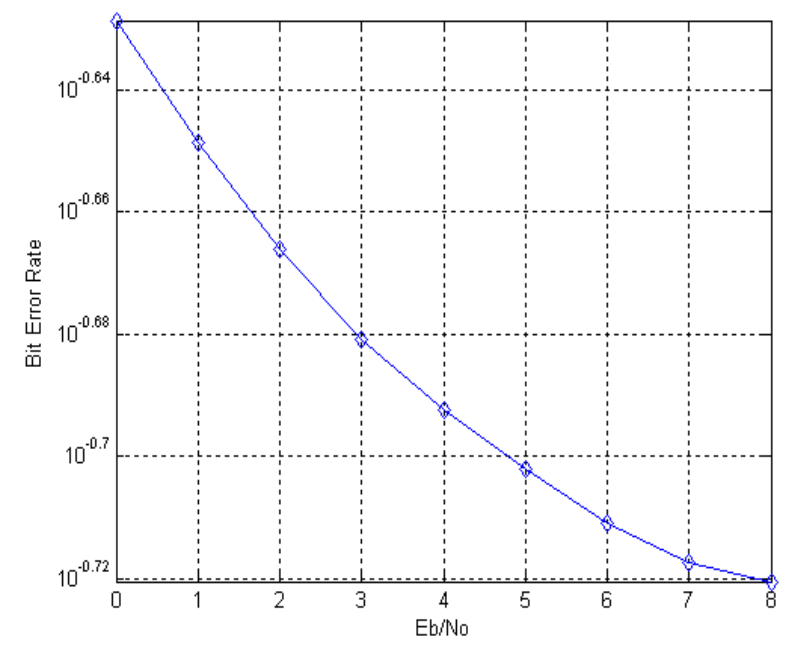

Figure-7: BER performance of 8-QAM for Rayleigh fading.

The Figure 4-7 show that in case of RAYLEIGH fading, the bit error rate (BER) is least for QAM modulation technique as compared to the other three modulation techniques i.e. BPSK, QPSK, 8PSK . Hence the performance of the Quadrature Amplitude Modulation is best when RAYLEIGH fading is present in 2:1MISO system.

\section{SIMULATION RESULTS OF SER FOR RAYLEINGH FADING WITH DIFFERENT MODULTION TECHNIQUES}

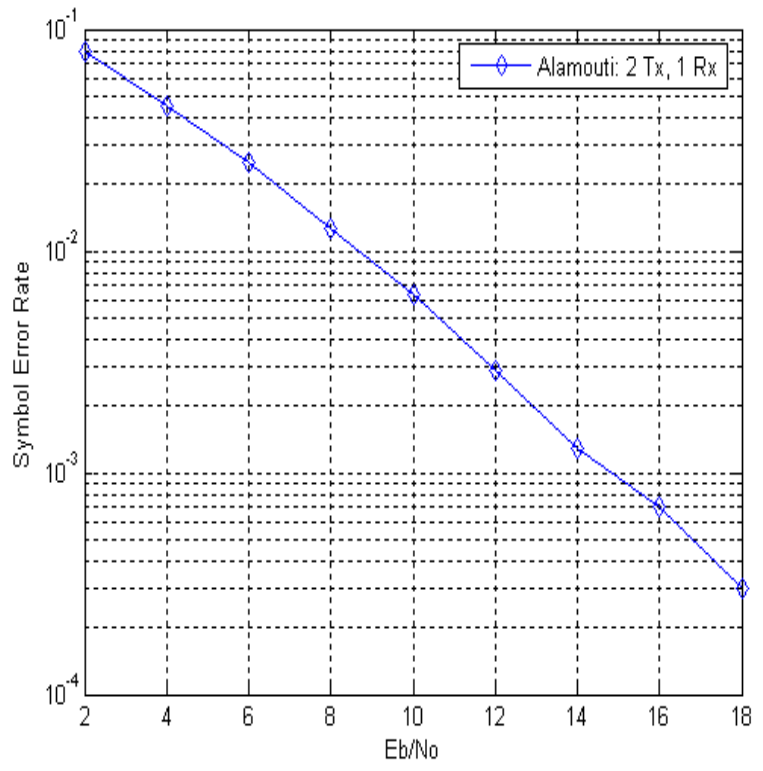

Figure-8: SER performance of BPSK for Rayleigh fading.

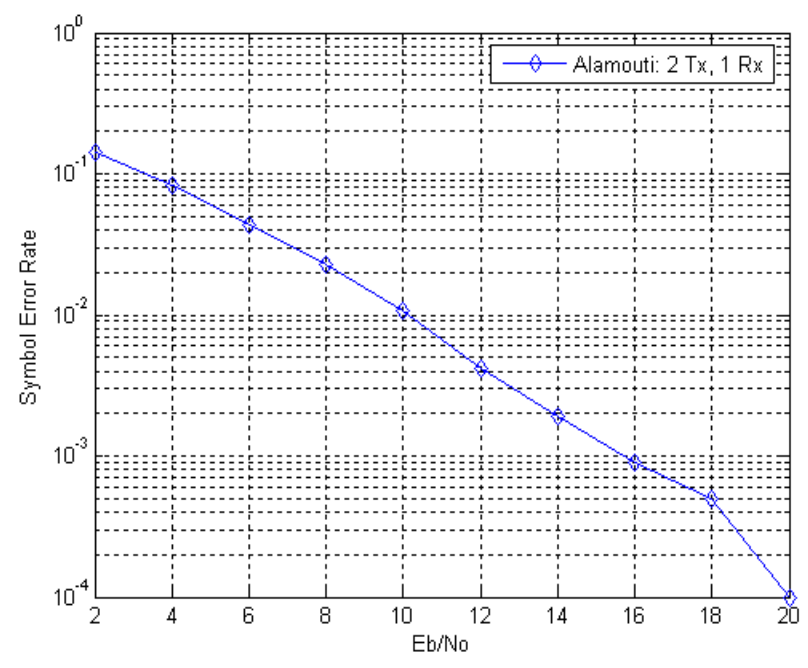

Figure-9: SER performance of QPSK for Rayleigh fading 


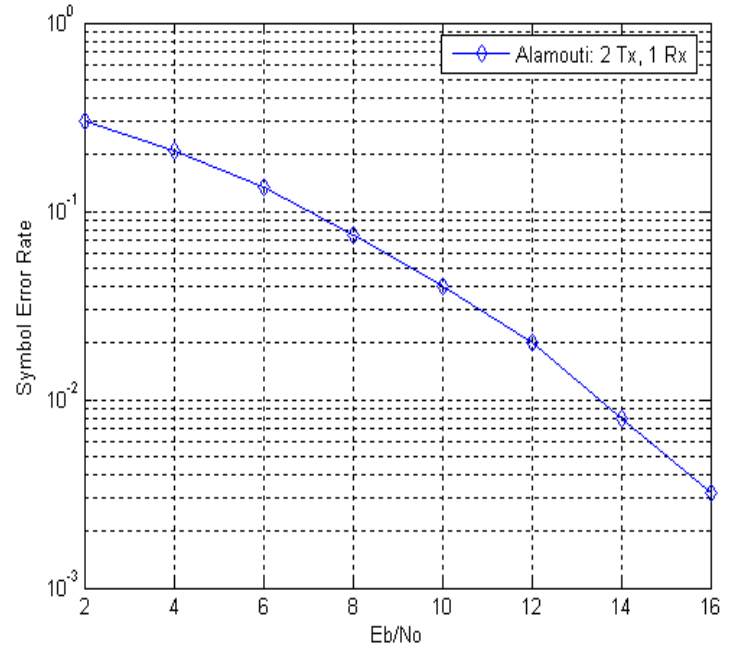

Figure-10: SER performance of 8-PSK for Rayleigh fading

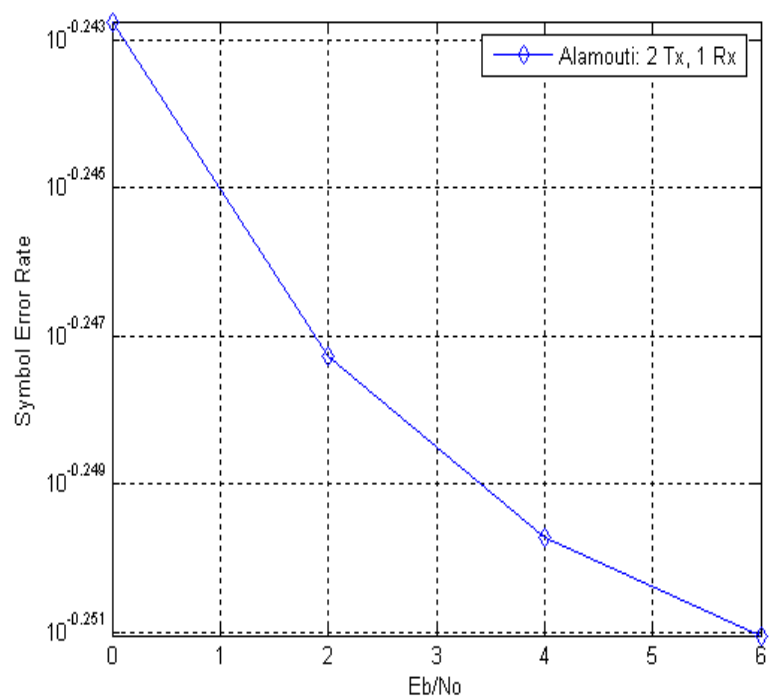

Figure-11: SER performance of 8-QAM for Rayleigh fading

The Figure 8-11 shows that in case of RAYLEIGH fading the symbol error rate(SER) is least for QAM modulation technique as compared to the other three modulation techniques i.e. BPSK, QPSK, 8PSK . Hence the performance of the Quadrature Amplitude Modulation is best when RAYLEIGH fading is present in 2:1 MISO system.

\section{COMPARISON OF VARIOUS MODULATIONS UNDER RAYLEIGH FADING}

Table-1 BER Performance under RAYLEIGH Fading at SNR of 8db

\begin{tabular}{|l|l|}
\hline Modulation Technique & Bit Error Rate \\
\hline BPSK & 0.031 \\
\hline
\end{tabular}

\begin{tabular}{|l|l|}
\hline QPSK & 0.01 \\
\hline 8-PSK & 0.0012 \\
\hline 8-QAM & 0.0010 \\
\hline
\end{tabular}

Table-1 shows that in case of 8-QAM modulation technique the value of bit error rate is least i.e.0.001 under RAYLEIGH fading environment as compared to other modulations. So, the performance of 8-QAM modulation technique is better in comparison to BPSK, QPSK and 8PSK.

Table-2 SER performance under RAYLEIGH fading at SNR of $6 \mathrm{db}$

\begin{tabular}{|l|l|}
\hline Modulation Technique & Symbol Error Rate(SER) \\
\hline BPSK & 0.015 \\
\hline QPSK & 0.014 \\
\hline 8-PSK & 0.002 \\
\hline 8-QAM & 0.001 \\
\hline
\end{tabular}

Table-2 shows that in case of 8-QAM modulation technique the value of symbol error rate (SER) is least i.e.0.001 under RAYLEIGH fading environment as compared to other modulations. So, the performance of 8-QAM modulation technique is better in comparison to BPSK, QPSK and 8PSK.

\section{CONCLUSION}

In this paper a comparative study of different modulation techniques with 2:1 MISO system under different Rayleigh fading environments is done. The figures 4-7 show that in case of RAYLEIGH fading channel the bit error rate (BER) is least i.e. 0.001 for 8-QAM modulation technique as compared to the other three modulation techniques i.e. BPSK, QPSK, 8PSK. Hence the performance of the Quadrature Amplitude Modulation is best when RAYLEIGH fading is present in 2:1 MISO system. It is also found that in case of RAYLEIGH fading the symbol error rate (SER) is least i.e. 0.001 for QAM modulation technique as compared to the other three modulation techniques i.e. BPSK, QPSK, 8PSK. [Figure 8-11]So, the performance of 8-QAM modulation technique is better in comparison to BPSK, QPSK and 8-PSK in terms of SER

\section{REFERENCES}

[1] N.Seshadri and J. H. Winters, "Two signaling schemes for improving the error performance of FDD transmission systems using transmitter antenna diversity," in Proc. 1993 IEEE Vehicular Technology Conf. (VTC 43rd), May 1993, pp. 508511. 
IOSR Journal of Engineering

Apr. 2012, Vol. 2(4) pp: 738-743

[2] J.G. Proakis, Digital Communications, New York: McGraw-Hill Inc., $3^{\text {rd }}$ Edition, 1995, pp. 758-833.

[3] A.Paulraj, Introduction to Space Time Communications, Prentice Hall of India, page 89, 93, 113

[4] M.Surendra Raju, A.Ramesh and A.Chockalingam,"BER Analysis of QAM with Transmit Diversity in Rayleigh Fading Channels", in IEEE 2003.

[5] Y N Trivedi and A K Chaturvedi, "Performance Analysis of Alamouti Scheme with Transmit Antenna Selection in MISO Systems", IEEE in 2010.

[6] Theodore. S. Rappaport, Wireless Communications, chapter 7 . 